\title{
On Fractional Schrödinger Equation
}

\author{
Piotr Rozmej ${ }^{1}$, Bartosz Bandrowski ${ }^{2}$ \\ ${ }^{1}$ Institute of Physics, ${ }^{2}$ Faculty of Mathematics Computer Science and Econometrics \\ University of Zielona Góra, ul. Szafrana 4a, 65-516 Zielona Góra, Poland \\ e-mail:P.Rozmej@if.uz.zgora.pl,B.Bandrowski@gmail.com
}

(Received: 23 March 2010; accepted: 7 July 2010; published online: 13 September 2010)

Abstract: In the note, recent efforts to derive fractional quantum mechanics are recalled. Some applications of a fractional approach to the Schrödinger equation are discussed as well.

Key words: Fractional calculus, fractional quantum mechanics, fractional Schrödinger equation

\section{INTRODUCTION}

The concept of fractional calculus, originated from Leibniz, has gained increasing interest during last two decades (see e.g. [1] and activity of the Fracalmo research group [2]). However, only during the last decade have scientific papers concerning fractional quantum mechanics appeared. Laskin [3] has shown that the path integral approach over Lévy paths leads to fractional quantum mechanics. Next, he considered the fractional Schrödinger equation for some particular cases like fractional Bohr atom and 1-dimensional fractional oscillator [4]. Some other cases of the fractional Schrödinger equation were discussed by Naber [5] and Ben Adda \& Cresson [6]. Recently Herrmann has applied the fractional mechanical approach to several particular problems [7-12].

Fractional calculus. The fractional calculus provides a set of axioms and methods to extend the coordinate and corresponding derivative definitions from integer $n$ to arbitrary order $\alpha,\left\{x^{n}, \partial^{n} / \partial x^{n}\right\} \rightarrow\left\{x^{\alpha}, \partial^{\alpha} / \partial x^{\alpha}\right\}$ in a reasonable way. The first question was already raised by Leibniz (1646-1716): Can we define a derivative of the order $1 / 2$, i.e. so that a double action of that derivative gives the ordinary one?

The definition of a fractional derivative is not unique; several definitions (the Riemann-Liouville, Caputo, Weyl, Riesz, Grünwald [1]) coexist.
The Riemann-Liouville fractional integral is defined as follows

$$
=\left\{\begin{array}{c}
\left({ }_{R} I^{\alpha} f(x)=\right. \\
\left({ }_{R}^{\alpha} f\right)(x)=\frac{1}{\Gamma(\alpha)} \int_{0}^{x} d \xi(x-\xi)^{\alpha-1} f(\xi)(x)=\frac{1}{\Gamma(\alpha)} \int_{0}^{x} d \xi(\xi-x)^{\alpha-1} f(\xi) \quad x<0 .
\end{array}\right.
$$

In (1) $\Gamma(z)$ is the Euler gamma function.

The Riemann-Liouville fractional derivative is defined as the result of fractional integration followed by the ordinary differentiation:

$$
\begin{gathered}
{ }_{R} D_{x}^{\alpha}=\frac{\partial}{\partial x}{ }_{R} I^{1-\alpha} . \\
= \begin{cases}{ }_{R} D_{x}^{\alpha} f(x)= \\
\left({ }_{R} D_{+}^{\alpha} f\right)(x)=\frac{1}{\Gamma(1-\alpha)} \frac{\partial}{\partial x} \int_{0}^{x} d \xi(x-\xi)^{-\alpha} f(\xi) & x \geq 0 \\
\left({ }_{R} D_{-}^{\alpha} f\right)(x)=\frac{1}{\Gamma(1-\alpha)} \frac{\partial}{\partial x} \int_{0}^{x} d \xi(\xi-x)^{-\alpha} f(\xi) & x<0 .\end{cases}
\end{gathered}
$$

The Caputo fractional derivative. The Caputo definition of the fractional derivative follows the inverted sequence of operations. An ordinary differentiation is followed by the fractional integration: 


$$
{ }_{C} D_{x}^{\alpha}={ }_{R} I^{1-\alpha} \frac{\partial}{\partial x}
$$

In the sequel we follow the Riemann-Liouville definition of the fractional derivative.

The fractional Schrödinger equation. The most general fractional Schrödinger equation may be obtained when

$$
\frac{\partial}{\partial t} \rightarrow \frac{\partial^{\alpha}}{\partial t^{\alpha}} \text { and } \frac{\partial}{\partial x} \rightarrow \frac{\partial^{\beta}}{\partial x^{\beta}} .
$$

However, special care has to be undertaken in order to introduce canonically conjugated observables $\hat{X}$ and $\hat{P}$, which are introduced as derivative operators on a Hilbert space of square integrable wave functions $f$. In standard $\mathrm{QM}$, the space coordinate representations of those operators are

$$
\hat{X} f(x)=x f(x), \quad \hat{P} f(x)=-i \hbar \partial_{x} f(x), \quad[\hat{X}, \hat{P}] f(x)=i \hbar .
$$

\section{SPACE FRACTIONAL SCHRÖDINGER EQUATION}

\section{Generalizations of operators $\hat{X}$ and $\hat{P}$ to the fractional order $[7,8]$}

Let us define

$$
\bar{\chi}:=\operatorname{sign}(x)|x|^{\alpha} \text { and } \bar{D}_{\bar{\chi}}:=\operatorname{sign}(x) D_{x}^{\alpha}(|x|) .
$$

For $f(\bar{\chi})=\bar{\chi}^{v}$ we obtain

$$
\bar{D}_{\bar{\chi}} \bar{\chi}^{v}=\frac{\Gamma(1+v \alpha)}{\Gamma(1+(v-1) \alpha)} \bar{\chi}^{v-1} .
$$

We are able to define Riemann-Taylor series $f(\bar{\chi})$ on $\mathbb{R}$

$$
f(\bar{\chi})=|\bar{\chi}|^{\frac{\alpha-1}{\alpha}} \sum_{n=0}^{\infty} a_{n} \bar{\chi}^{n}
$$

With the integral operator $\int_{|-x|}^{|x|} d \bar{u}=\operatorname{sign}(x) I_{x}^{\alpha}(|x|)$ one defines the fractional scalar product

$$
\langle f \mid g\rangle=\int_{-|x|}^{|x|} d \bar{u} f *(\bar{\chi}) g(\bar{\chi})
$$

With these definitions the fractional observables $\hat{X}$ and $\hat{P}$ read as

$$
\begin{aligned}
& \hat{X} f(\bar{\chi})=\left(\frac{\hbar}{m c}\right)^{(1-\alpha)} \bar{\chi} f(\bar{\chi}) \\
& \text { and } \hat{P} f(\bar{\chi})=-i\left(\frac{\hbar}{m c}\right)^{\alpha} m c \bar{D}_{\bar{\chi}} f(\bar{\chi}) .
\end{aligned}
$$

For a function set $\left\{f(\bar{\chi})=\bar{\chi}^{v}\right\}$ the commutation relation takes the following form

$$
[\hat{X}, \hat{P}]=-i \hbar c(v, \alpha),
$$

where

$$
c(v, \alpha)=\frac{\Gamma(1+v \alpha)}{\Gamma(1+(v-1) \alpha)}-\frac{\Gamma(1+(v+1) \alpha)}{\Gamma(1+v \alpha)} .
$$

The above definitions may be extended to a multidimensional case, for $N$ particles, $i=1, \ldots, 3 N$

$$
\begin{aligned}
& \left\{\hat{X}_{i}\right\}=\left\{\left(\frac{\hbar}{m c}\right)^{1-\alpha} \bar{\chi}_{i}\right\}, \\
& \left\{\hat{P}_{i}\right\}=\left\{-i\left(\frac{\hbar}{m c}\right)^{\alpha} m c \bar{D}_{\bar{\chi}}\right\} .
\end{aligned}
$$

On a function set of the form

$$
\left\{f\left(\bar{\chi}_{1}, \ldots, \bar{\chi}_{3 N}\right)=\prod_{i}^{3 N} \bar{\chi}_{i}^{v_{i}}\right\}
$$

one obtains

$$
\begin{aligned}
& {\left[\hat{X}_{i}, \hat{X}_{j}\right]=\left[\hat{P}_{i}, \hat{P}_{j}\right]=0,} \\
& {\left[\hat{X}_{i}, \hat{P}_{j}\right]=-i \hbar \delta_{i j} c\left(v_{i}, \alpha\right),}
\end{aligned}
$$

where

$$
c\left(v_{i}, \alpha\right)=\frac{\Gamma\left(1+v_{i} \alpha\right)}{\Gamma\left(1+\left(v_{i}-1\right) \alpha\right)}-\frac{\Gamma\left(1+\left(v_{i}+1\right) \alpha\right)}{\Gamma\left(1+v_{i} \alpha\right)} .
$$

\section{Angular momentum operators and eigenvalues}

In [8], R. Herrmann defines angular momentum-like operators

$$
L_{i j}=\hat{X}_{i} \hat{P}_{j}-\hat{X}_{j}, \hat{P}_{i}=-i \hbar\left(\bar{\chi}_{i} \bar{D}_{j}-\bar{\chi}_{j} \bar{D}_{i}\right) .
$$

Their commutation relations are isomorphic to an extended fractional $S O^{\alpha}(3 N)$ algebra

$$
\begin{gathered}
{\left[L_{i j}, L_{m n}\right]=} \\
=i \hbar c(v, \alpha)\left(\delta_{i m} L_{j n}+\delta_{j n} L_{i m}-\delta_{i n} L_{j m}-\delta_{j m} L_{i n}\right) .
\end{gathered}
$$

Casimir operators $\Lambda_{k}^{2}$ associated with $S O^{\alpha}(k)$ may be defined as

$$
\Lambda_{k}^{2}=\frac{1}{2} \sum_{i, j}^{k}\left(L_{i, j}\right)^{2}, \quad k=2, \ldots, 3 N .
$$

They fulfill the following commutation relations: 


$$
\left[\Lambda_{3 N}^{2}, L_{i j}\right]=0 \text { and }\left[\Lambda_{k}^{2}, \Lambda_{k^{\prime}}^{2}\right]=0 .
$$

For $(N=1)$ one has and $\hat{J}_{z}(\alpha)=\Lambda_{2}=L_{12}$ and $\hat{J}^{2}(\alpha)=\Lambda_{2}=L_{3}^{2}=L_{12}^{2}+L_{13}^{2}+L_{23}^{2}$. Then, one can find solutions for eigenvalue equations for fractional case

$$
\begin{aligned}
\hat{J}_{z}(\alpha)|J M\rangle_{f} & =\hbar \frac{\Gamma(1+M \alpha)}{\Gamma(1+(J-1) \alpha)}|J M\rangle_{f}, \\
M & =0, \pm 1, \pm 2, \ldots, \pm J, \\
\hat{J}^{2}(\alpha)|J M\rangle_{f} & =\hbar^{2} \frac{\Gamma(1+(J+1) \alpha)}{\Gamma(1+(J-1) \alpha)}|J M\rangle_{f}, \\
J & =0,+1,+2, \ldots,
\end{aligned}
$$

which for $\alpha=1$ coincide with the usual angular momentum eigenfunctions and eigenvalues $\left(|J M\rangle_{f}(\alpha=1) \rightarrow|J M\rangle\right)$.

\section{SOME RESULTS}

Applications of fractional quantum mechanics to some particular problems have been done up to now only by R. Herrmann [7-12]. In [7] the author shows that charmonium spectrum may be understood quantitatively in the framework of (space) fractional Schrödinger equation with $\alpha \approx 2 / 3$.

In [8], he shows that measured energies of rotational nuclear states may be reproduced by the fractional symmetric rigid rotor model. Rotational, $\gamma$-unstable and vibrational character of spectra is obtained for different values of $\alpha: 1 \geq \alpha \geq 0.8,0.8 \geq \alpha \geq 0.6,0.6 \geq \alpha \geq 0.4$, respectively (see Fig. 5 from [8]). In [9, 10] R. Herrmann emphasizes the close relation between fractional calculus and $q$-deformad Lie algebras and discusses the principle of local gauge invariance for fractional fields.

In [11] the author constructs nuclear single particle Hamiltonian from fractional rotational groups $S O^{\alpha}$ (3) and then applies it as a basis to calculate the microscopic part of the total nuclear energy within macroscopic-microscopic approach. The model reproduces magic numbers and for spherical nuclei and can be extended for deformed nuclei. That potential used in calculations of nuclear masses (binding energies) approach performs quite well (see Fig. 7 from [11]). The similar approach has been used in [12] for obtaining electronic magic numbers in metal clusters.

\section{Time fractional Schrödinger equation}

The general idea is to replace $\partial / \partial t \rightarrow \partial^{\alpha} / \partial t^{\alpha}$ or $i \hbar \partial / \partial t \rightarrow(i \hbar \partial / \partial t)^{\alpha}$ keeping the position and momentum operators in the usual form. M. Naber [5] gives some physical arguments in favour of the latter case. He considers the time fractional Schrödinger equation for a free particle and a particle in a potential well and obtains solutions in terms of Mittag-Leffler functions and functions related to them. For the free particle case the wave function evolves in such a way that the total probability increases with time and for $t \rightarrow \infty$ reaches the limiting value $1 / \alpha^{2}$. The same result was obtained in the case of the particle in a potential well. However, the energy levels for the potential are time dependent. All those results apply to the case with $\alpha \in(0,1]$. It is worth mentioning that though the qualitative properties of the time evolution are known, the details are hidden in hardly computable special functions (Mittag-Leffler, Fox, Wright etc.).

Recently, working on some other problems, we have developed a numerical method for solving integral equations of convolution type [13]. Such integral equations appeared to be (under some assumptions concerning boundary conditions) equivalent to differential evolution equations with fractional time but with a fractional derivative of the order $\alpha \in[1,2]$. Below we present the first results obtained for the time fractional Schrödinger equation with $\alpha \in[1,2]$ for a free particle. The method is applicable to a particle in a potential depending smoothly on coordinates, as well. In our numerical exercise we simplified the fractional Schrödinger equation to the following form:

$$
\left(i \frac{\partial}{\partial t}\right)^{\alpha} \psi(x, t)=-\nabla^{2} \psi(x, t) .
$$

The time evolution of the free particle Gaussian wave packet according to the equation (15) is presented in Fig. 1. The presented results correspond to fractional order $\alpha=1.02$.

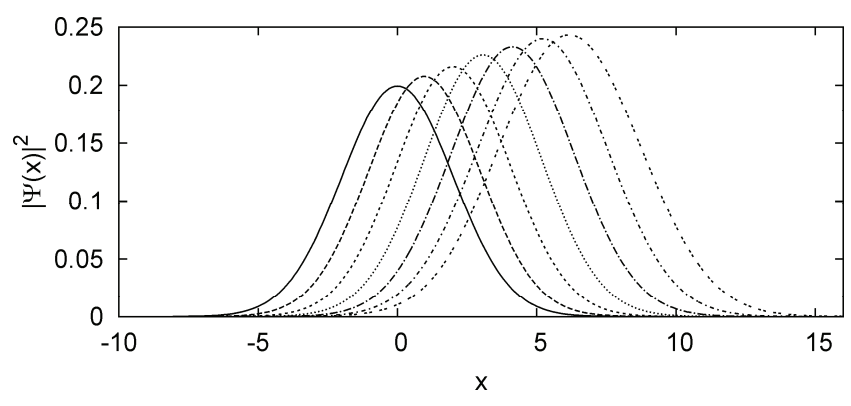

Fig. 1. $|\psi(x)|^{2}(15)$ as function of the position for time instants $t=0,1,2,3,4,5$ and 6

The initial wave function is

$$
\psi(x, t=0)=(2 \pi)^{-1 / 4} \sigma^{-1 / 2} \exp \left[-\frac{\left(x-x_{0}\right)^{2}}{4 \sigma^{2}}+i p_{0}\left(x-x_{0}\right)\right],
$$


where $x_{0}=0, p_{0}=1$ and $\sigma=2$. The details of the wave packet motion (real and imaginary parts of $\psi$ ) are similar to the case of an ordinary Schrödinger equation. However, it is seen that the total probability increases with time. For bigger values of $\alpha$ the probability increases much faster.

In conclusion, we want to emphasize that though the basis of fractional quantum mechanics is already formulated, the range of solved problems is very narrow. Therefore, it can be considered as an interesting field for further exploration.

\section{References}

[1] I. Podlubny, Fractional Differential Equations. Academic Press, 1999.

[2] Fractional Calculus Modeling, http://www.fracalmo.org/

[3] N. Laskin, Fractional quantum mechanics and Lévy path integrals. Phys. Lett. A 268, 298 (2000); Fractional quantum mechanics. Phys. Rev. E 62, 3135 (2000).

[4] N. Laskin, Fractional Schrödinger equation. Phys. Rev. E 66, 056108 (2002).
[5] M. Naber, Time fractional Schrödinger equation. J. Math. Phys. 45, 3339 (2004).

[6] F. Ben Adda, J. Cresson, Fractional differential equations and the Schrödinger equation. Appl. Math. Comp. 161, 323 (2005).

[7] R. Herrmann, Properties of fractional derivative Schrödinger type wave equation and a new interpretation of the charmonium spectrum. arXiv:math-ph/05100099 (2006).

[8] R. Herrmann, The fractional symmetric rigid rotor. J. Phys. G 34, 607 (2007).

[9] R. Herrmann, q-deformed Lie algebras and fractional calculus. arXiv:0711:3701 (2007).

[10] R. Herrmann, Gauge invariance in fractional field theories. Phys. Lett. A 372, 5515 (2008)

[11] R. Herrmann, Fractional dynamic symmetries and the ground properties of nuclei. arXiv:0806.2300 (2008).

[12] R. Herrmann, Fractional phase transition in medium size metal cluster and some remarks on magic numners in gravitationally and weakly interacting clusters. arXiv:0907.1953 (2009).

[13] B. Bandrowski, A. Karczewska, P. Rozmej, Numerical solutions to integral equations equivalent to differential equations with fractional time derivative. Int. J. Appl. Math. Comp. Sci. 20 (2), 261-269 (2010). (http://www.uz.zgora.pl/ prozmej/amcs2.pdf)

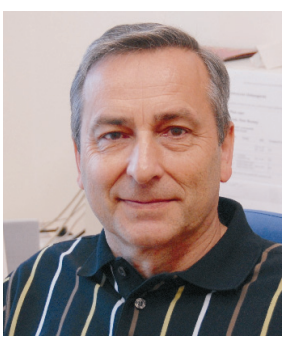

Piotr RozMEJ is professor at the Faculty of Physics and Astronomy, University of Zielona Góra, head of the Division of Mathematical Methods in Physics. He holds an M.Sc. degree from University MCS, Lublin, a Ph.D. degree from Warsaw University and habilitation from UMCS. Full professor since 1997. He works in the fields of nuclear structure, nuclear reactions, properties of heavy and superheavy nuclei. The other fields of his activity contain quantum optics, in particular wave packet dynamics, nonlinear dynamics, deterministic chaos and numerical methods.

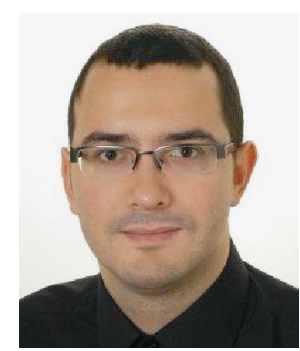

BARTOSZ BANDrowski is a Ph.D. student at the Faculty of Mathematics, Computer Science and Econometrics, University of Zielona Góra. He received an M.Sc. degree in mathematics from the University of Zielona Góra in 2008. His main interests include mathematical informatics, numerical methods in Volterra equations, fractional calculus and stochastic equations. 